\title{
Oral health and covid19
}

\author{
Camila Lindoni Azevedo ${ }^{1}$, Edgard Michel Crosato ${ }^{1}$, Isabella da Cunha Henriques ${ }^{2}$ and Paulo Sérgio Gomes Henriques ${ }^{3 *}$ \\ ${ }^{1}$ Universidade de São Paulo Faculdade de Odontologia: Sao Paulo, SP, Brazil \\ ${ }^{2}$ Albert Einstein Israeli Faculty of Health Sciences, Sao Paulo, SP, Brazil \\ ${ }^{3}$ São Leopoldo Mandic, Institute and Dental Research Center, Campinas, SP Brazil
}

\section{Introduction}

Since December 2019, the newly discovered coronavirus (2019nCov) has caused an outbreak of pneumonia in Wuhan and throughout China. 2019-nCov enters host cells through human cell receptor ACE2, the same with SARS-CoV, but with higher binding affinity. The rapidly increasing number of cases and evidence of human-tohuman transmission suggested that the virus was more contagious than SARS-CoV and MERS-CoV. By mid-February 2020, a large number of infections of medical staff have been reported, and the specific reasons for protection failure needs to be further investigated. This new infectious agent is more likely to affect older males and causes severe respiratory diseases indicating that a new person-to-person transmission infectious agent has caused this emergent viral pneumonia outbreak. Although clinics such as stomatology have been closed during the epidemic, a large number of emergency patients still go to the dental clinics and hospitals for treatment. We have summarized the possible transmission routes of 2019-nCov in stomatology, such as the airborne spread, contact spread, and contaminated surface spread. We also reviewed several detailed practical strategies to block virus transmission to provide a reference for preventing the transmission of 2019-nCov during dental diagnosis and treatment, including patient evaluation, hand hygiene, personal protective measures for the dental professionals, mouthrinse before dental procedures, rubber dam isolation, anti-retraction handpiece, disinfection of the clinic settings, and management of medical waste [1].

Clinical gingival inflammation is a well-defined site-specific condition for which several measurement systems have been proposed and validated, and epidemiological studies consistently indicate its high prevalence globally. Gingivitis is generally regarded as a site-specific inflammatory condition initiated by dental biofilm accumulation and characterized by gingival redness and edema and the absence of periodontal attachment loss. When compared to periodontitis, a peculiarity of plaque-induced gingivitis is the complete reversibility of the tissue alterations once the dental biofilm is removed [2]. Various, studies have suggested a relationship between psychosocial factors and periodontal disease. That can be explained by behavioural changes and host-immune responses. Stress, anxiety and depression can provoke this modification [3].

Notwithstanding the reversibility of the gingivitis elicited tissue changes, gingivitis holds particular clinical significance because it is considered the precursor of periodontitis, a disease characterized by gingival inflammation combined with connective tissue attachment and bone loss. Periodontitis is chronic inflammatory disease, initiated by the accumulation of a pathogenic dental plaque biofilm above and below the gingival (gum) margin, and within which microbial dysbiosis leads to a chronic non-resolving and destructive inflammatory response. It has a prevalence of $45-50 \%$ in adults in its mildest form rising to over $60 \%$ in people over 65 years of age $64 \%$. Severe periodontitis is estimated to affect $11.2 \%$ of the global adult population and is a major cause of tooth loss [4].

It should come as no surprise to the reader that the oral cavity, including the periodontal tissues, are not somehow disjointed from the rest of the body [5].

In recent decades there has been a renewed interest in the fundamental principle that the oral microbiota might play an important role in systemic health. Specifically, the presence of an oral infection such as periodontal disease might be a causative or exacerbating factor of some systemic conditions. Accounts emphasizing the importance of oral health and its influence in systemic health date back to ancient history and have accompanied the evolution of science and medicine throughout time. Therefore, the idea of a link between oral and systemic health is not new. These oral bacteria and their products (antigens and endotoxins, among others) can enter the blood stream or respiratory tract resulting in a typically transient bacteremia that can trigger complications for immunocompromised and otherwise susceptible individuals [6].

\section{Diabetes mellitus}

Periodontal disease and diabetes mellitus present a highly complex relationship often characterized as bidirectional that has been extensively studied over recent decades with several epidemiological and experimental studies. They have demonstrated that diabetes mellitus is a risk factor for periodontal disease, that periodontal disease is more prevalent and severe in diabetic patients, but also that the inflammatory mechanisms of periodontal diseases can adversely affect metabolic control of diabetes, playing a role in its pathogene-sis and its complications [6].

Little evidence is available regarding the effects a long-term periodontal infection on diabetes control. This study evaluates the influence of periodontal status on changes of glycated hemoglobin (HbAlc) levels of type 2 diabetics and showed. The periodontitis progression was associated with increases in HbAlc in Type 2 diabetics and suggests that periodontal treatment may improve glycemic control by eliminating periodontal infeccion [7].

\section{Respiratory diseases}

Poor oral health (periodontal disease and caries) has been associated with respiratory diseases such as chronic obstructive

${ }^{*}$ Correspondence to: Paulo Sérgio Gomes Henriques, Rua Hilário Magro Jr. 37 Campinas, São Paulo, Brazil, Zip Code 13026.123, E-mail: contato@periotech.com.br

Received: April 01, 2020; Accepted: April 14, 2020; Published: April 17, 2020 
pulmonary disease (COPD) and bacterial pneumonia, with several subtypes such as nosocomial (hospital-acquired) pneumonia, nursing home-associated pneumonia, aspiration pneumonia and ventilatorassociated pneumonia. Several mechanisms have been proposed linking oral bacteria to these respiratory diseases, like the aspiration of oral and respiratory pathogens colonizing oral tissues, and inflammatory cytokines from periodontal tissues affecting respiratory epithelium and promoting infection. The association between periodontal disease and pneumonia presents overall a stronger level of evidence and poor oral hygiene and periodontal disease were associated with the colonization of oral tissues by respiratory pathogens and that diverse oral treatment and interventions promoting better plaque control resulted in a $40 \%$ reduction in the incidence of nosocomial (hospital-acquired) pneumonia [6].

\section{Cardiovascular diseases}

There is a strong association in literature between poor oral health and acute myocardial infarction independent of classical cardiovascular disease risk factors. Atherosclerotic vascular disease is the main cause of cardiovascular disease, and the possible association of atherosclerosis and periodontal disease has been the target of several longitudinal and cross-sectional studies, evidencing that patients with cardiovascular problems have a tendency to a worse periodontal status. Periodontal disease triggers a systemic inflammatory response which leads to high levels of various cytokines, also associated with atherosclerotic vascular disease, such as interleukin-1beta, interleukin-6, interleukin-8, tumor necrosis factor alpha, and the potential pathogens can gain access to the circulatory system, resulting in a state of typical transient bacteremia. The resulting bacteremia is more severe in patients with gingivitis or periodontal disease and can either directly influence cardiovascular disease. Overall, the current data suggests that periodontal treatment might result in a reduction of cardiovascular disease risk achieved by the control of biomarkers associated with cardiovascular disease events (inflammatory and thrombotic markers, adhesion molecules and vascular function) [6].

Thus, the proposition is that the control of periodontal diseases, via adequate treatment, can improve the overall health of the patient by reducing the burden of aggression on the organism.

\section{Smoking}

Epidemiologic studies have revealed that smoking is one of the major lifestyle-related environmental risk factors for periodontal disease. Both the local and systemic effects of cigarette smoke should be in- trinsically considered. Inhaled cigarette smoke is absorbed from the capillary vessels via the pulmonary alveolar epithelium and enters the systemic circulation, whereas direct exposure of inhaled cigarette smoke to periodontal tissues causes vasoconstriction of the periodontal microvasculature and gingival fibrosis, which is often observed in smokers. Although plaque accumulation and disease progression are exacerbated in smokers, smokers have fewer clinical signs and symptoms of gingival inflammation, and therefore smoking can mask an underlying gingivitis [8].

Smoking cessation was the most important measure in the management of periodontitis, after the removal of the bacterial biofilm [9].

\section{Dental plaque control}

Dental plaque is the principal etiologic factor in the development of periodontal diseases. A mechanical plaque control program design by dental professionals could help patients achieve excellent oral hygiene, oral health and overall health [10].
Despite that, periodontal disease are highly prevalent globally. The accumulation of dental plaque and calculus is usually caused by improper toothbrushing techniques, failure to carry out interdental cleaning and irregular dental visits [11].

Health behaviors are strongly related to flexibility and control in the daily routines. Toothcleaning behaviors are part of daily routines that must include toothbrushing, interdental brushing and adjunctive antiseptics in mouthrinses and dentifrices [12]. Based on two systematic reviews, there is good evidence that improved oral hygiene and frequent professional oral health care can reduce the progression or occurrence of respiratory diseases among high-risk elderly adults $[13,14]$, which are more related with comorbidities and poorer clinical COVID-19 outcomes [15].

Bass technique is the most accepted and effective method for plaque removal and should be done for at least two minutes twice a day. There is no method of toothbrushing to be clearly superior to others [16]. Plaque removal can be more effective with eletric toothbrushes than manual, and should be recommended mostly for less motivated person or those with disabilities [17].

Toothbrushing alone is able to clean only the buccal, lingual and occlusal tooth surface, although to prevent and reduce the incidence of caries and periodontal disease, must put a major focus on the interdental and proximal areas of the dentition [18]. The results indicate that interdental brushes are superior to waxed dental floss in interproximal plaque removal and it is important that the size of the interdental brush match the interdental area [19].

Flossing cannot be recommended other than for sites of gingival and periodontal health, where interdental brushes will not pass through the interproximal area without trauma. In patients with gingivitis once daily interdental cleaning is recommended and the adjunctive use of chemical plaque control agents offers advantages [20].

Brushing is the most practiced oral hygiene method for plaque removal. The prevalence of plaque associated with periodontal disease in adults aged 35 to 44 years is $99 \%$ for gingivitis and up to $52.7 \%$ for periodontitis.

Brushing only is not sufficient for removing plaque, especially at the gingival margin and interproximal region Compared with dental floss, the use of an interdental brush showed greater efficacy in controlling the interproximal biofilm around teeth and dental implants. Thus, we must educate and encourage our patients to use these specific methods of interdental cleaning on a daily basis for effective biofilm control [21].

Psychosocial factors were also associated with oral health riskrelated behaviors such as tobacco smoking and plaque levels. Emotional status may directly affect host resistance factors as well as lead to poorer oral hygiene, smoking and use of alcohol, and changes in diet and exercise [22].

\section{Exercise habits}

Periodontal diseases are closely related to lifestyle and obesity. Moderate exercise habits contributes to improvement on this situation. A pathology that combines a lifestyle-related disease and obesity is called metabolic syndrome that is identified by symptoms including abdominal obesity, dyslipidemia, hyperglycemia, and hypertension. It is also widely known that moderate exercise habits lead to improvement in lifestyle-related diseases. Some reports suggested that moderate exercise routinely alleviates systemic inflammatory reactions and 
that a decrease in the amount of physical activity increases the risk of many chronic diseases. In general, habitual exercise activates local immunocompetent cells such as macrophages, neutrophils, and lymphocytes, been moderate exercise beneficial immunologically. Therefore, habitual exercise may contribute not only to improvement in periodontal disease but also to improvement in the pathology of lifestyle-related diseases [23].

\section{Conclusion}

At this moment, April 20, 2020, the WHO total counts individual around the world is: ACTIVE 1.621.394, DEATHS: $166.053(6,85 \%)$, RECOVERIES: 636.187(26.25\%).

Blood glucose control, quit smoking, practice physical exercises, and decrease the plaque index through a rigorous daily oral hygiene program with brushing, flossing, interdental brushes and tongue cleaning after main meals, will represent in this delicate moment of the pandemic(mainly in older individuals and comorbidities like diabetes, cardiovascular and respiratory diseases), an important immunological differential that can save lives.

This reinforces the major rule of preventive oral health care and health promotion that should be implemented by dentists and related professionals.

\section{References}

1. Peng X, Xu X, Li Y, Cheng L, Zhou X, et al. (2020) Transmission routes of 2019-nCoV and controls in dental practice. Int J Oral Sci 12: 9.

2. Trombelli L, Farina R, Silva CO, Tatakis DN (2018) Plaque-induced gingivitis: Case definition and diagnostic considerations. J Periodontol 89: 46-47.

3. Cakmak,O, Tasdemir Z, Aral CA, Dundar S, Koca HB (2016) Gingival crevicular fluid and saliva stress hormone levels in patients with chronic and aggressive periodontitis. J Clin Periodontol 43: 1024-1031.

4. Sanz M, Ceriello A, Buysschaert M, Chapple I, Demmer RT, et al. (2018) Scientific evidence on the links between periodontal diseases and diabetes: Consensus report and guidelines of the joint workshop on periodontal diseases and diabetes by the International diabetes Federation and the European Federation of Periodontology. Diabetes Res Clin Prac 137: 231-241.

5. Sharma P (2020) An Update on the Links Between Periodontal Health and General Health. Prim Dent J 8: 22-27.

6. Falcao A, Bullón P (2019) A review of the influence of periodontal treatment in systemic diseases. Periodontol 2000 79: 117-128.
7. Costa KL, Taboza ZA, Angelino GB, Silveira VR, Montenegro R Jr, et al. (2016) The influence of periodontal disease on changes of glycated hemoglobin levels in type 2 diabetics: a retrospective cohort study. J Periodontol 26: 1-13.

8. Murakami S, Mealey BL, Mariotti A, Chapple IC (2018) Dental plaque-induced gingival conditions. J Clin Periodontol 45: 17-27.

9. Ramseier CA (2005) Potential impact of subject-based risk factor control on periodontitis. J Clin Periodontol. 2005; 32(6):283-290.

10. World Health Organization (1978) Epidemiology, Etiology, and Prevention of Periodontal Disease. WHO Technical Report, Series 621, Geneva.

11. Lertpimonchai A, Rattanasiri S, Arj-Ong Vallibhakara S, Attia J, Thakkinstian A (2017) The association between oral hygiene and periodontitis: a systematic review and metaanalysis. Int Dent J 67: 332-343.

12. Figuero E, Roldán S, Serrano J, Escribano M, Martín C,et al. (2019) Efficacy of Adjunctive Therapies in Patients With Gingival Inflammation. A Systematic Review and Meta-Analysis. J Clin Periodontol.

13. Pedersen PU, Larsen P, Ha ${ }^{\circ}$ konsen SJ (2016) The effectiveness of systematic perioperative oral hygiene in reduction of postoperative respiratory tract infections after elective thoracic surgery in adults: a systematic review. JBI Database System Rev Implement Rep 14: 140-173.

14. Azarpazhooh A, Leake JL (2006) Systematic review of the association between respiratory disease and oral health. J Periodontol 77: 1465-1482.

15. Guan WJ, Liang WH, Zhao Y, Liang HR, Chen ZS (2020) China Medical Treatment Expert Group for Covid-19. Comorbidity and its impact on 1590 patients with Covid-19 in China: A Nationwide Analysis. Eur Respir J 26: 2000547

16. Joshi S, Suominen AL, Knuuttila M, Bernabé E (2018) Toothbrushing behaviour and periodontal pocketing: An 11-year longitudinal study. J Clin Periodontol 45: 196-203.

17. van der Weijden FA, Timmerman MF, Piscaer M, IJzerman Y, Warren PR, et al. (1998). A comparison of the efficacy of a novel electric toothbrush and a manual toothbrush in the treatment of gingivitis. Am J Dent 11: 23-28.

18. Löe H (2000) Oral hygiene in the prevention of caries and periodontal disease. Int Dent J 50: 129-139.

19. Bergenholtz, Olsson (1984) Efficacy of plaque removal using interdental brushes and waxed dental floss. Scand J Dent Res 92: 198-203.

20. Chapple IL, Van der Weijden F, Doerfer C, Herrera D, Shapira L, et al. (2015) Primary prevention of periodontitis: managing gingivitis. J Clin Periodontol 42: 71-76.

21. Luz M, Klingbeil MFG, Henriques PSG, Lewgoy HR (2016) Comparison between interdental brush and dental floss for controlling interproximal biofilm in teeth and implants. Dent Health Curr 1-4.

22. Sheiham A, Nicolau B (2005) Evaluation of social and psychological factors in periodontal disease. Periodontol 2000 39: 118-131.

23. Omori S, Uchida F, Oh S, So R, Tsujimoto T, et al. (2018) Exercise habituation is effective for improvement of periodontal disease status: a prospective intervention study. Therapeutics Clin Risk Management 14: 565-574.

Copyright: (C2020 Azevedo CL. This is an open-access article distributed under the terms of the Creative Commons Attribution License, which permits unrestricted use, distribution, and reproduction in any medium, provided the original author and source are credited. 